\title{
1 Pan-cancer analysis of mRNA stability for decoding tumour post-transcriptional
}

\section{2 programs}

3 Gabrielle Perron ${ }^{1,2}$, Pouria Jandaghi ${ }^{2}$, Maryam Rajaee ${ }^{2}$, Rached Alkallas ${ }^{1,2}$, Yasser Riazalhosseini ${ }^{1,2}$,

$4 \quad$ Hamed S. Najafabadi ${ }^{1,2, *}$

5

61 Department of Human Genetics, McGill University, Montreal, QC H3A 1B1, Canada

72 McGill Genome Centre, Montreal, QC H3A 0G1, Canada

8

9 * Corresponding author: H. S. Najafabadi, hamed.najafabadi@mcgill.ca

10 


\section{Abstract}

12 RNA stability is a crucial and often overlooked determinant of gene expression. Some of the regulators of

13 mRNA stability are long known as key oncogenic or tumour suppressor factors. Nonetheless, the extent to

14 which mRNA stability contributes to transcriptome remodeling in cancer is unknown, and the factors that

15 modulate mRNA stability during cancer development and progression are largely uncharacterized. Here,

16 by decoupling transcriptional and post-transcriptional effects in RNA-seq data of 7760 samples from 18

17 cancer types, we present a pan-cancer view of the mRNA stability changes that accompany tumour

18 development and progression. We show that thousands of genes are dysregulated at the mRNA stability

19 level, and identify the potential factors that drive these changes, including $>80$ RNA-binding proteins

20 (RBPs) and microRNAs (miRNAs). Most RBPs and miRNAs have cancer type-specific activities, but a

21 few show recurrent inactivation across multiple cancers, including the RBFOX family of RBPs and miR-

22 29. Analysis of cell lines with phenotypic activation or inhibition of RBFOX1 and miR-29 confirms their

23 role in modulation of genes that are dysregulated across multiple cancers, with functions in calcium

24 signaling, extracellular matrix organization, and stemness. Overall, our study highlights the critical role of 25 mRNA stability in shaping the tumour transcriptome, with recurrent post-transcriptional changes that are

$26 \sim 30 \%$ as frequent as transcriptional events. These results provide a resource for systematic interrogation

27 of cancer-associated stability drivers and pathways.

29 Keywords: RNA stability, gene regulation, cancer, microRNAs, RNA-binding proteins 


\section{Background}

Widespread disruption of gene expression programs is a hallmark of cancer and underlies the extensive transformation of tumour cell identity and behavior. Among the least understood aspects of this gene expression remodeling is the regulation of mRNA stability and decay. Previous studies have found specific programs that are involved in tumorigenesis or metastasis through modulation of mRNA stability [1-8]; however, the extent to which mRNA stability contributes to cancer cell transcriptome has not been systematically studied, and the associated regulatory networks are mostly unknown. A key limitation in studying these post-transcriptional programs stems simply from our lack of ability to measure mRNA decay rate in vivo: traditional methods that measure mRNA decay rely on in vitro manipulations such as transcriptional inhibition with chemical inhibitors (e.g. actinomycin D) or metabolic labeling with nucleoside analogues (e.g. 4-thiouridine), combined with time series measurements of transcripts [9-11]. Despite recent improvements [12,13], these methods are resource-intensive, have inherent limitations and biases such as triggering cellular stress and pleiotropic effects [14], and, most importantly, are only applicable to in vitro models. As a result, the mRNA stability landscape of tumour remains almost completely uncharted across different cancer types.

A potential solution comes from recent studies showing that tissue RNA-seq data contain enough information to disentangle transcription rate from mRNA decay rate. Briefly, under the assumption that RNA processing rate is constant $[15,16]$, any change in unspliced (pre-mature) mRNA abundance

49 (estimated from intronic reads) must reflect a proportional change in transcription rate, while any change in spliced (mature) mRNA abundance (estimated from exonic reads) reflects the combined effect of transcription rate and mRNA decay (Fig. 1a). This model enables the estimation of differential mRNA stability based on how the ratio of exonic and intronic reads changes across conditions [15]. A recent improvement on this model generalizes the unspliced-spliced relationship as a power-law function, with

54 the power-law exponent reflecting the coupling between transcription rate and splicing rate [17] (Fig.

S1a,b). 
Here, we build on these methods to obtain a pan-cancer map of mRNA stability changes between

tumour and normal tissues, as well as the mRNA stability changes that accompany tumour progression.

To do so, we first introduce a general framework for statistical analysis of differential mRNA stability that takes into account the distributional properties of count data. We benchmark this method using experimental measurements of mRNA decay rate, and then apply it to the RNA-seq data from The Cancer Genome Atlas (TCGA) to map the mRNA stability landscapes of 18 cancer types. We identify thousands of transcripts whose stability is altered during tumour formation and/or progression - experimental measurements in cancer cell line models support these findings and suggest a role for mRNA stability alterations in tumor progression and invasiveness. Finally, using network modeling and functional experiments, we identify key microRNAs (miRNAs) and RNA-binding proteins (RBPs) that mediate these changes, providing new insights into the post-transcriptional mechanisms of transcriptome remodelling in cancer.

\section{Results}

\section{A generalized linear model for statistical testing of mRNA stability}

The spliced and unspliced transcripts of each gene follow a power-law relationship, with deviations from this power-law trend reflecting changes in the degradation rate of the mature mRNA [17] (Fig. S1a,b). The power-law exponent reflects the coupling between transcription rate and RNA processing rate - an exponent of 1 indicates no coupling between transcription and processing rate constants, whereas values smaller than 1 indicate that as transcription increases, processing rate constant decreases, potentially due to saturation of the RNA processing machinery (Fig. S1a). To use this power-law relationship for the inference of differential stability, it is essential to correctly model the variability in RNA-seq counts. For this purpose, we developed DiffRAC (https://github.com/csglab/DiffRAC), a framework that converts the unspliced-spliced relationship to a generalized linear model whose parameters can then be inferred from sequencing count data using an appropriate error model of choice

(Fig. 1b-c and Fig. S1 c-d). 
We evaluated the performance of DiffRAC for estimating differential mRNA stability using a previously published benchmarking dataset [18], consisting of RNA-seq data from mouse embryonic stem cells and terminal neurons, along with ground-truth transcript half-life measurements after transcriptional blockage with actinomycin D. We observed a modest overall correlation between RNAseq-based stability estimates from DiffRAC and ground-truth stability measurements (Pearson correlation 0.22; Fig. 1d and Table S1), in line with previous reports on RNA stability estimation using this specific benchmarking dataset $[17,18]$. However, for genes that had narrow confidence intervals as estimated by DiffRAC, the Pearson correlation between RNA-seq-based estimates and ground truth exceeded 0.5 (Fig. 1d-f), indicating that the confidence intervals estimated by DiffRAC indeed reflect the true uncertainty in estimating differential mRNA stability. Based on (adjusted) P-values associated with DiffRAC differential stability estimates, we identified 79 genes with higher stability in embryonic stem cells and 37 genes with higher stability in terminally differentiated neurons (FDR $<0.05)$, which closely correspond to differentially stable genes based on the ground truth (Fig. 1g). Overall, these results suggest that DiffRAC can properly estimate not just the mean differential mRNA stability, but also its uncertainty and statistical significance.

One limitation of the model described above is that, with increasing sample sizes, the number of latent variables that need to be estimated by regression also increases, which can become prohibitively expensive in terms of computational times. To overcome the challenges associated with fitting the model in large sample cohorts, we developed a simplified DiffRAC model that assumes most of the variance in transcription can be explained by the experimental variables (see Methods and Fig. S2a-c). This assumption greatly reduces the number of parameters; however, we observed that it does not significantly alter the differential stability estimates in the benchmarking dataset (Fig. S2d).

\section{DiffRAC identifies cancer-associated changes in mRNA stability}

To investigate the post-transcriptional changes responsible for transcriptome remodeling in cancer, we performed a pan-cancer analysis of differential mRNA stability across TCGA, encompassing 7760 
samples from 18 cancer types. We used DiffRAC to identify genes that were differentially stabilized or destabilized in tumour compared to normal tissues in each cancer type. This analysis revealed an average of 3954 genes that were differentially stabilized/destabilized per cancer type (FDR-adjusted $\mathrm{p}<0.05$ )

(Fig. 2a-b, Fig. S3, and Table S2), suggesting widespread post-transcriptional remodeling in cancer. The majority of genes showed highly cancer-specific stability profiles (Fig. 2b).

Several lines of evidence support the reliability of the stability profiles we have inferred. First, we observed that tumour mRNA stability profiles clustered by organ of origin (Fig. 2 b), providing an internal validation for the robustness of stability inferences. Secondly, we observed that post-transcriptionally deregulated genes in each cancer type are functionally related (Fig. 2c), consistent with previously reported relationship between post-transcriptional regulons and functional gene modules $[19,20]$. This analysis also highlights the role of mRNA stability in shaping the functional landscape of the cancer cell. For example, epithelial-mesenchymal transition genes and MYC targets are enriched among stabilized genes across several cancer types, while metabolic pathways such as oxidative phosphorylation and lipid metabolism are highly enriched among destabilized genes most noticeably in cholangiocarcinoma (CHOL), liver hepatocellular carcinoma (LIHC) and head-neck squamous cell carcinoma (HNSC). Thirdly, we found that cancer-associated stability changes inferred from tissue RNA-seq data are highly consistent with experimentally measured mRNA stability changes in cancer cell line models. Specifically, we used time-series measurements of 4-thiouridine-labeled RNA [21] from MDA-MB-231 cell line, a model of breast cancer, as well as the highly invasive MDA-LM2 cells to identify mRNAs that are differentially stable between these two cell lines (see Methods for details; measurements are provided in Table S3). We found that the mRNAs that are more stable in breast tumours compared to normal tissue (based on DiffRAC analysis of tissue RNA-seq data) are also overall more stable in the highly invasive LM2 line compared to the parental MDA line (based on experimental stability measurements). Similarly, tumour-destabilized mRNAs that DiffRAC identified are overall less stable in the LM2 line (Fig. 2d). 
Since the MDA-LM2 line is more invasive than MDA-MB-231, the above analysis also suggests

133 that, at least in breast cancer, normal-to-tumour stability changes persist during the progression of the

134 disease. In fact, two of the three pathways that were upregulated in breast tumors (Fig. 2c) also appear to

135 be enriched among mRNAs that are stabilized in MDA-LM2 compared to MDA-MB-231 cell lines

136 (MYC targets and mTORC1 signaling, Fig 2e; example genes are shown in Fig. 2f), supporting a role of

137 mRNA stability in deregulation of these key pathways during both cancer development and progression.

138 To understand whether normal-to-tumour stability changes are correlated with progression-associated

139 stability changes across other cancers, we used DiffRAC to examine tumour stage and grade in each

140 TCGA cancer type, while controlling for the confounding effects of age, sex, ancestry, and tumour purity

141 (Table S4). We identified a total of 1966 genes with significant stability changes associated with tumor

142 stage in at least one of the 11 cancers types that we analyzed (Table S5), and 2013 genes whose stability

143 was associated with tumor grade in at least one of the four cancer types for which this type of

144 classification was available (Table S6). We observed highly cancer-specific associations both for stage

145 and grade (Fig. 2g). Importantly, we found that in most cases the stage- and grade-associated stability

146 changes correlate with normal-to-tumour stability changes (Fig. 2h shows an example, with the overall

147 results summarized in Fig. 2i). Taken together, these results highlight widespread mRNA stability

148 changes in tumours, which affect key cancer-related pathways. This post-transcriptional deregulation

149 continues to remodel the transcriptome through cancer progression, as evident from analysis of stage- and

150 grade-associated stability changes.

RNA-binding proteins play a key role in shaping the tumour mRNA stability profile

154 stability. These sequence-specific factors primarily affect RNA stability through binding to the 3'

155 untranslated region (UTR) of their targets - RBPs either stabilize or destabilize their targets [22], while

156 miRNAs primarily destabilize their target mRNAs $[23,24]$. Starting with RBPs, we set out to examine

157 whether these factors underlie the mRNA stability changes in cancer. We specifically tested for the 
enrichment of the targets of each RBP among genes that are differentially stable between tumour and normal tissues, after correcting for the background frequency of RBP binding to each gene (see

160 Methods). Figure 3a shows an example, where the binding targets of the RBFOX1 protein are enriched 161 among genes that are destabilized in glioblastoma multiforme (GBM), relative to the binding targets of 162 other RBPs. We can quantify this enrichment by statistical modeling of the relationship between the 163 binding of a specific RBP to the 3' UTR of a gene and the tumour-specific stability status of that gene

164 (Fig. 3b). We performed a systematic quantification of these relationships for 35 RBPs whose stability 165 target sets (regulons) have been previously mapped based on the presence of their preferred binding 166 sequences in the 3' UTRs as well as the expression pattern of the candidate target genes [22]. This 167 analysis revealed significantly enriched regulons among tumour-stabilized or destabilized genes across 168 different cancer types, representing deregulation of 17 out of the 35 examined RBPs in at least one cancer 169 type (Fig. 3c). Importantly, we observed excellent agreement between cancer-associated RBP expression 170 changes and RBP target enrichments, after taking into account the expected function of each RBP in 171 stabilizing or destabilizing its targets (Pearson correlation 0.61; Fig 3d). For example, SNRPA, which is 172 an RNA-destabilizing factor [22], is up-regulated in multiple cancers, consistent with the observed 173 destabilization of its regulon (Fig. 3c-d). This strong correlation highlights the reliability of our regulon 174 analysis approach for identifying dysregulated RBPs, and suggests that aberrant expression of RBPs in 175 cancer drives coordinated changes in the stability of their regulons.

Among the RBPs we analyzed, two RBPs, namely RBFOX1 and RBFOX3, stand out as being 177 consistently deregulated across several cancer types. Specifically, the targets of these RBPs are enriched 178 among destabilized genes in almost half of all the cancer types we analyzed (Fig. 3c). Consistent with the 179 role of RBFOX proteins in promoting mRNA stability $[22,25]$, both RBFOX1 and RBFOX3 are down180 regulated across multiple cancers (Fig. 4a-b), suggesting that down-regulation of RBFOX proteins leads 181 to destabilization of their targets. For both RBFOX1 and RBFOX3, the highest expression in normal

182 tissues can be seen in the brain tissue; subsequently, the most prominent case of their down-regulation as 183 well as the most significant changes in the stability of their regulons can be seen in GBM, suggesting a 
major role in determining tumour transcriptome in this cancer type. However, their effect is not limited to GBM, especially for RBFOX3, which shows a broader range of expression in normal tissues and is also downregulated in a greater number of cancers (Fig. $\mathbf{4 b})$.

To confirm that the down-regulation of RBFOX proteins accompanies destabilization of their direct binding targets in cancer, we used HITS-CLIP data of RBFOX proteins in neurons [26] to build a highconfidence stability network, including genes that have the strongest binding sites in their 3' UTRs (see Methods). As expected, we observed overall destabilization of HITS-CLIP-based RBFOX targets across different cancers (Fig. 4c). We noticed a subset of genes that are consistently destabilized across the same cancers in which either RBFOX1 or RBFOX3 is downregulated (Fig 4c). Interestingly, a subgroup of these genes is stabilized in the few cancer types in which RBFOX1 is upregulated (e.g. genes with positive stability values for LUSC, LUAD and THCA in Fig 4c), further supporting the notion that their cancer-associated stability changes are driven by RBFOX proteins.

To verify that the stability of these genes is regulated by RBFOX1, we examined the RNA-seq data from differentiated primary human neural progenitor (PHNP) cells in which RBFOX1 is knocked down [27]. As expected, cancer-destabilized genes that were associated with RBFOX1 were also downregulated upon RBFOX1 knockdown (Table S7 and Fig. 4d). In contrast, when RBFOX1 expression is restored ectopically in the mouse neurons lacking RBFOX proteins [25], the expression of these genes is also rescued (Fig. 4e). We identified a core set of eight genes that have RBFOX binding site in their 3' UTRs, are concurrently destabilized across cancers, are inhibited when RBFOX1 is knocked down, and are upregulated when RBFOX1 expression is rescued (Fig. 4f). Interestingly, half of these genes belong to the calcium signaling pathway (based on KEGG pathways [28], Fisher's exact test $\mathrm{P}<10^{-6}$ ), suggesting that deregulation of RBFOX proteins primarily affects calcium signaling in cancer cells.

\section{Dysregulation of miRNA regulons shapes the cancer transcriptome}

To examine the contribution of miRNAs to the dysregulation of mRNA stability in cancer, we systematically searched for miRNAs whose targets are disproportionately dysregulated at the stability 
210 level in cancer, similar to the RBP analysis above (Methods). Fig. 5a shows miR-122 as an example;

211 miR-122 is the most abundant miRNA expressed in liver cells [29], was previously shown to be

212 downregulated in cholangiocarcinoma, and acts as a tumor suppressor via suppression of cell proliferation

213 and induction of apoptosis [30,31]. As expected, our regulon analysis indicates that miR-122 targets are

214 predominantly stabilized specifically in cholangiocarcinoma tumours compared to normal tissue (Fig.

215 5a), consistent with reduced activity of miR-122. This observation is consistent with TCGA miRNA

216 expression data, which show specific downregulation of miR-122 expression in cholangiocarcinoma (Fig.

217 S4). Systematic application of this network-based approach revealed that, out of 153 broadly conserved

218 miRNA families, the regulons of 63 miRNAs are deregulated in at least one cancer type, suggesting

219 widespread disruption of miRNA networks (Fig. 5b).

Of interest, we observed that miR-29 targets are recurrently stabilized across more than half of the

cancer types we analyzed, suggesting a pan-cancer decrease in miR-29 activity. Among these cancer

230 genes in cancer, while restoring its activity partially rescues the normal mRNA stability landscape of the

231 cell.

\section{Discussion}

By quantifying differential mRNA stability patterns across 18 cancer types, our study presents a 
uncovered recurrent changes in the stability of $>13,000$ genes in at least one cancer type, highlighting the widespread role of post-transcriptional regulation in shaping the cancer transcriptome. We note that this resource also provides an approximation for the relative contribution of transcriptional and posttranscriptional events in shaping cancer transcriptome: on average, $19 \%$ of genes that are significantly up-

240 regulated at the expression level are detected by DiffRAC as significantly stabilized in tumour, and $23 \%$

241 of genes with significantly reduced expression are detected as significantly destabilized. In comparison,

$24266 \%$ and $61 \%$ of genes whose expression is significantly up- or down-regulated are detected as

243 transcriptionally activated or inhibited in tumour, respectively (Fig. S6). These results suggest a

244 significant role for post-transcriptional changes in shaping the cancer transcriptome, with recurrent

245 changes that are $\sim 30 \%$ as frequent as transcriptional events.

Our study also highlights the coordinated post-transcriptional deregulation of genes that are

247 involved in the same pathways. Notably, we observed recurrent stabilization of mRNAs that encode

248 epithelial-mesenchymal transition proteins and MYC targets across multiple cancer types. The epithelial-

249 mesenchymal transition (EMT) is the process by which epithelial cells lose their apical-basal polarity and

250 cell-cell adhesion, and instead acquire mesenchymal properties such as migratory and invasive potentials

251 [32]; our results suggest that activation of the EMT pathway in cancer is at least partly mediated by post-

252 transcriptional up-regulation. Similarly, we observed post-transcriptional up-regulation of MYC targets,

253 which include growth-related genes that directly contribute to tumorigenesis [33]. MYC is a well-defined

254 transcription factor and represents one of the most frequently amplified oncogenes [34], leading to

255 transcriptional activation of its targets in cancer. Therefore, our intriguing observation that MYC targets

256 are also up-regulated at the mRNA stability level suggests the presence of convergent transcriptional and

257 post-transcriptional mechanisms that modulate overlapping gene sets. Furthermore, we observed

258 coordinated destabilization of mRNAs for genes implicated in oxidative phosphorylation (OXPHOS) and

259 related pathways such as fatty acid metabolism and adipogenesis, consistent with the well-documented

260 Warburg effect in which upregulation of glucose consumption and glycolysis is accompanied by a down-

261 regulation of OXPHOS [35]. 
In addition, we observed widespread and coordinated post-transcriptional modulation of the targets of RNA-binding proteins (RBPs) in cancer, with the RBFOX family of RBPs standing out as having the most recurrently down-regulated regulon across multiple cancer types. RBFOX proteins are known

265 regulators of alternative splicing and mRNA stability [22] and have been implicated in a number of 266 neurological diseases $[17,25,36]$, but their role in cancer is less characterized. Nonetheless, at least the

267 RBFOX1 locus appears to be among the most frequently deleted loci across different cancer types [37,

268 38], with its deletion [39] or specific genetic variations [40] associated with poor survival. Our study

269 suggests that downregulation of RBFOX proteins leads to destabilization of their target transcripts in tumour; many of these transcripts encode proteins involved in calcium signaling, a critical pathway that

271 affects a wide range of cancer-associated processes such as proliferation, invasion, and apoptosis [41].

272 The association between RBFOX1 and calcium signaling is also supported by previous literature that

273 shows a positive effect of RBFOX1 on the expression of some of the genes involved in this pathway [42].

277 in several cancer types [43, 44], affecting cell proliferation, differentiation and apoptosis [45]. This

278 downregulation correlates with more aggressive forms of cancer, characterized by increased metastasis,

279 invasion and relapse [46], and therapeutic restoration of miR-29 was suggested to improve disease

280 prognosis [47]. In line with these reports, we observed pan-cancer stabilization of miR-29 targets,

281 suggesting widespread reduction in miR-29 activity in cancer, which could be partially reversed by miR-

28229 rescue. We note that our results highlight a core set of 64 genes that are bound by miR-29, stabilized at

283 least in KIRC, and down-regulated after restoring miR-29 activity in the KIRC model cell line 786-O

284 (Fig. 5e). Importantly, eight of these genes are markers of embryonal carcinoma, suggesting that miR-29

285 inhibition is essential for activation of an embryonic-like program in cancer. In addition, we observed a

286 significant enrichment of the extracellular matrix (ECM) genes (Fig. 5e), suggesting that miR-29 
inhibition also contributes to ECM remodeling in cancer, consistent with previous reports on ECM regulation by miR-29 [48].

Together, these results highlight a key role for mRNA stability programs, mediated by RBPs and miRNAs, in regulation of pathways that are integral to cancer development and progression. While the

291 vast majority of current literature is focused on the role of transcriptional mechanisms in reprogramming

292 cancer cells, this study underlines a critical and largely uncharacterized role for post-transcriptional

293 remodeling of the cancer cell transcriptome, and provides a resource for exploring post-transcriptional

294 pathways in cancer.

295

296

\section{Methods}

\section{Joint modelling of intronic and exonic read counts and mRNA stability}

Our approach for statistical modeling of intronic and exonic read counts builds on previous research that connects the abundance of pre-mRNA and mature mRNA to mRNA stability (Fig. S1a-b):

$$
\log \boldsymbol{m}=b \times \log \boldsymbol{p}+\log \varphi+\log \gamma
$$

Here, $m$ corresponds to the vector of the mature mRNA abundance for a given gene across different samples, $p$ is the abundance of the pre-mature mRNA, $\gamma$ is the mRNA stability across samples, $\varphi$ is the maximum processing rate of RNA, and $b$ is the bias-term (Fig. S1b). Vectors are differentiated from scalars using bold typeface. We further model the logarithm of mRNA stability as a linear function of a set of sample-level variables:

$$
\log \gamma=\boldsymbol{X} \times \boldsymbol{\beta}+\alpha
$$

Here, $X$ is the $n \times k$ matrix of sample-level variables (for $n$ samples and $k$ variables), $\beta$ is the vector of coefficients that quantify the effect of each variable on the mRNA stability, and $\alpha$ is an intercept (matrices are differentiated from vectors using capital letters). This leads to:

$$
\log \boldsymbol{m}=b \times \log \boldsymbol{p}+c+\boldsymbol{X} \times \boldsymbol{\beta}
$$


311 where $c=\log \varphi+\alpha$. We model the mean of intronic read counts for a given gene across samples as a

312 function of the pre-mRNA abundance for that gene, a gene-level scaling factor that can be interpreted as

313 the effective length, and a sample-specific scaling factor that can be interpreted as library size:

$$
\lambda^{i n t}=\boldsymbol{p} \times l \times \boldsymbol{s}^{i n t}
$$

315 Here, int stands for intronic, $\lambda$ represents the mean read count, $l$ is the gene-specific scaling factor, and $s$ is

316 the sample-specific scaling factor. Similarly, the mean of exonic read counts for a given gene across

317 samples can be expressed as:

$$
\lambda^{\text {exo }}=\boldsymbol{m} \times l^{\prime} \times \boldsymbol{s}^{\text {exo }}
$$

319 The above equations can be collectively expressed by matrix operations as:

$$
\log \left[\begin{array}{l}
\lambda^{i n t} \\
\lambda^{\text {exo }}
\end{array}\right]=\log \left[\begin{array}{c}
\boldsymbol{s}^{i n t} \\
\boldsymbol{s}^{\text {exo }}
\end{array}\right]+\boldsymbol{X}^{\prime} \times\left[\begin{array}{c}
\log \boldsymbol{p}^{\prime} \\
c^{\prime} \\
\boldsymbol{\beta}
\end{array}\right]
$$

321 where

$$
\boldsymbol{X}^{\prime}=\left[\begin{array}{ccc}
\boldsymbol{I}_{n} & \mathbf{0}_{n \times 1} & \mathbf{0}_{n \times k} \\
b \times \boldsymbol{I}_{n} & \mathbf{1}_{n \times 1} & \boldsymbol{X}_{n \times k}
\end{array}\right]
$$

323 and $p^{\prime}=p \times l, c^{\prime}=c+\log \left(l^{\prime} / l\right)$, and $I$ is the identity matrix (matrix dimensions are indicated as

324 subscripts). These equations connect pre-/mature mRNA abundance and mRNA stability to the observed

325 intronic and exonic read counts for each given gene (see Fig. S1c-d for matrix equations that consider all

326 genes at the same time). This formulation enables the estimation of unknown parameters using a

327 generalized linear model with a log-link function. In this study, we use DESeq2 [49] to fit the unknown

328 parameters of this model, as explained below.

\section{Estimation of the effect of sample variables on mRNA stability}

331 The above equations allow us to estimate the distribution of latent variables $\log p^{\prime}, c^{\prime}$, and $\beta$ by fitting the

332 model to observed intronic and exonic read counts. For this purpose, we use the matrix $X^{\prime}$ as the design

333 matrix in a DESeq2 model. In practice, we replace the first column of $X^{\prime}$ with an intercept (Fig. 1c), 
334 which is an equivalent design matrix and does not change the interpretation of $\beta$, but enables the user to

335 employ a beta prior (if desired) when fitting the DESeq2 model.

In order to be able to construct $X^{\prime}$, the bias term $b$ needs to be first estimated. We do this by first

337 optimizing $b$ in order to maximize the likelihood of observed intronic and exonic read counts across all

338 genes in a model that assumes the mRNA stability is a gene-specific constant. Specifically, we use the

339 below design matrix $D$ to fit the model using DESeq2, while varying the value of $b$ in the interval $[0,1]$ to

340 select the $b$ that maximizes the sum of log-likelihood of the data across all genes:

$$
\boldsymbol{D}^{\prime}=\left[\begin{array}{cc}
\boldsymbol{I}_{n} & \mathbf{0}_{n \times 1} \\
b \times \boldsymbol{I}_{n} & \mathbf{1}_{n \times 1}
\end{array}\right]
$$

342 We use the 'optimize' function in R to select the optimal value of $b$. Once this optimal value is identified,

343 it is used in the matrix $X^{\prime}$ (see above), which is then used as the design matrix in DESeq2 to estimate the

344 latent variables, including $\beta$ (i.e. the effect of each variable on stability). This procedure is implemented in

345 DiffRAC (https://github.com/csglab/DiffRAC).

347 A modified design to accommodate larger sample sizes

348 A major limitation of this approach is the considerable increase in computing time with larger sample

349 sizes when DESeq2 is used to fit the model, since the model includes sample-specific latent variables for

350 pre-mRNA abundance. To accommodate these cases, we have also implemented a model that assumes

351 that most of the variance in pre-mRNA abundance can be explained by the experimental variables, instead

352 of including sample-specific latent variables:

$$
\log \boldsymbol{p}=\boldsymbol{X} \times \boldsymbol{\omega}+\rho
$$

354 Here, $\omega$ is the vector of coefficients that represent the effect of each variable on the pre-mRNA

355 abundance of a given gene, and $\rho$ is a gene-specific intercept. There, we also have:

$$
\log \boldsymbol{m}=b \times(\boldsymbol{X} \times \boldsymbol{\omega}+\rho)+c+\boldsymbol{X} \times \boldsymbol{\beta}
$$

357 This leads to a modified set of matrix equations (Fig. S2a-c) that connect intronic/exonic read counts to 358 sample variables: 


$$
\log \left[\begin{array}{l}
\lambda^{i n t} \\
\lambda^{\text {exo }}
\end{array}\right]=\log \left[\begin{array}{l}
\boldsymbol{s}^{\text {int }} \\
\boldsymbol{s}^{\text {exo }}
\end{array}\right]+\boldsymbol{X}^{\prime} \times\left[\begin{array}{c}
\rho^{\prime} \\
\boldsymbol{\omega} \\
c^{\prime} \\
\boldsymbol{\beta}
\end{array}\right]
$$

360 where

$$
\boldsymbol{X}^{\prime}=\left[\begin{array}{cccc}
\mathbf{1}_{n \times 1} & \boldsymbol{X}_{n \times k} & \mathbf{0}_{n \times 1} & \mathbf{0}_{n \times k} \\
\mathbf{1}_{n \times 1} & b \times \boldsymbol{X}_{n \times k} & \mathbf{1}_{n \times 1} & \boldsymbol{X}_{n \times k}
\end{array}\right]
$$

362 and $\rho^{\prime}=\rho+\log l$, and $c^{\prime}=c+\log \left(l^{\prime} / l\right)+\rho \times(\mathrm{b}-1)$. Similar to the previous section, $X^{\prime}$ can be used as the

363 design matrix for DESeq2 to estimate the latent variables, including $\omega$ and $\beta$. To construct $X^{\prime}$, the bias-

364 term $b$ is chosen so that it maximizes the sum of log-likelihood of data across all genes in a model that

365 assumes gene-specific constant stability, i.e. with the below design matrix $D^{\prime}$ :

$$
\boldsymbol{D}^{\prime}=\left[\begin{array}{ccc}
\mathbf{1}_{n \times 1} & \boldsymbol{X}_{n \times k} & \mathbf{0}_{n \times 1} \\
\mathbf{1}_{n \times 1} & b \times \boldsymbol{X}_{n \times k} & \mathbf{1}_{n \times 1}
\end{array}\right]
$$

367 This simplified model is also implemented in DiffRAC. Overall, we see strong agreement between

368 DiffRAC's estimates when using the two different models (i.e. sample-specific pre-mRNA abundances

369 vs. condition-specific pre-mRNA abundances) on the same data (Fig. S2d).

\section{TCGA RNA-seq data processing}

372 RNA-seq BAM files for 7078 tumor samples and 682 adjacent normal samples from the 18 cancer types

373 with at least 5 normal samples in TCGA were acquired from the National Cancer Institute (NCI) Genomic

374 Data Commons (GDC) data portal (https://portal.gdc.cancer.gov/GDC; dbGaP study accession

375 phs000178.v1.p1). In order to quantify the number of reads corresponding to pre-mRNA and mature

376 mRNA for the estimation of mRNA stability, we generated custom annotations for exons and introns for

377 the transcripts supported by both Ensembl and Havana consortia, using GTF formatted annotations

378 acquired from Ensembl GRCh38 version 87. To avoid the potential confounding effect of alternative

379 splicing on mature mRNA quantification, we exclusively retained exonic reads mapping to constitutive

380 exons that are present in all Ensembl/Havana transcripts. Intronic regions were included in our

381 annotations only if they were not overlapped by any exon, regardless of whether the exon was 
concordantly annotated by Ensembl or Havana consortia. The strandedness of RNA-seq data was determined using RSeQC [50]. Subsequently, BAM files were sorted by read name using SAMtools, and exonic and intronic reads were separately counted using HTSeq-count [51], limiting to reads with a

385 MAPQ score $\geq 30$. Exonic reads were counted using the HTSeq "intersection-strict" mode, whereas intronic reads were counted using the "union" mode. The exonic/intronic read counts were then used as input to DiffRAC for stability analysis. We removed the cell cycle genes (based on GO term GO:000704) for downstream analyses, given that these genes are not at steady state, which is required for estimating stability from pre-/mature mRNA abundances. When intersecting the DiffRAC KIRC results with miR-29 mimic expression results, however, we kept potential cell cycle genes that were significantly altered by

391 miR-29 mimic expression, as their differential expression is supported by direct measurements in isogenic 392 cell lines.

\section{Pathway analysis}

395 MSigDB hallmark gene-sets [52] were retrieved using the msigdbr R package (https://cran.r-

396 project.org/web/packages/msigdbr/index.html). For each TCGA cancer type, Fisher's exact test was used 397 to examine the association between each pathway and the sets of significantly stabilized or destabilized 398 genes, separately.

\section{Differential RNA stability between MDA-MB-231 and MDA-LM2 cells}

401 Raw RNA-seq reads for time-series measurements of 4-thiouridine (4sU)-labeled RNA [21] from MDA-

402 MB-231 and MDA-LM2 cells were obtained from GEO accession GSE49608 (SRA accession

403 SRP028570). This RNA-seq dataset represents time points 0, 2, 4, and 7h after a 2-hour treatment of cells

404 with 4sU (four replicates for each cell line at each time point). Reads were mapped to the GRCh38

405 genome assembly using HISAT2 [53], and gene-level read counts for each sample were obtained using

406 HTSeq-count [51] ("intersection-strict" mode) based on Ensembl GRCh38 v87 gene annotations.

407 Differential mRNA stability between the MDA-MB-231 and MDA-LM2 cells were obtained using 
DESeq2 [49] by modeling the RNA abundances as a function of $\sim c+t+c: t$, where $c$ is the cell type (0 for MDA-MB-231 and 1 for MDA-LM2), $t$ is the time point, and $c: t$ is the interaction between cell type and time. In this model, the coefficient of $c$ would represent the differential expression between the two

411 cell types (i.e. difference in abundance at time zero); the coefficient of $t$ would represent the stability of 412 each gene in the reference cell line MDA-MB-231 (relative to the average of all genes); and the 413 coefficient of the interaction term $c: t$ would represent the differential stability of each gene between the 414 two cell lines. For each gene, the coefficient of $c: t$ and associated statistics were retrieved using DESeq2.

\section{$\mathrm{RBP}$ and miRNA regulon analysis}

417 The stability regulons of 35 RBPs (i.e. the set of genes bound and regulated by each RBP) were obtained from a previous publication [22]. The regulons of miRNA families were obtained by identifying exact miRNA seed matches in the mRNA 3' UTRs. Specifically, 3' UTR sequences of protein-coding genes were retrieved using the Ensembl GRCh38 version 87 annotations. We limited the analysis to the genes for which a single 3' UTR, composed of a single exon, was shared across all isoforms, in order to avoid the possible confounding effects of alternative splicing. The miRNA seed sequences (8nt) were retrieved from TargetScan v7.2 [54], limiting to a set of 153 broadly conserved miRNA families (family conservation score $\geq 1$ ). Exact seed sequence matches in 3' UTR sequences were identified while limiting the search space to a maximum of $2000 \mathrm{nt}$ downstream of the stop codon.

427 The regulon enrichment among up-regulated or down-regulated genes was quantified using a logistic 428 regression approach. Specifically, for each cancer type, we modeled the likelihood of being bound by 429 each RBP/miRNA as a function of the gene status, with -1 corresponding to significantly destabilized 430 genes $(\mathrm{FDR} \leq 0.05),+1$ corresponding to significantly stabilized genes, and 0 corresponding to non-

431 significant genes. To account for the confounding factors that generally affect the number of binding sites 
number of binding sites of each gene for RBPs or miRNAs as the background. Specifically, we used a

434 generalized linear model of the binomial family, in which the presence of a binding site for the specific

435 RBP or miRNA of interest is considered as "success", and the presence of binding sites for other RBPs or

436 miRNAs considered as "failures". These success/failure counts were modeled as a function of the stability

437 status of the gene using the glm function in R.

\section{Cell culture and transient transfection of miRNA mimics}

440 The established renal cancer cell line 786-O was purchased from the American Type Culture Collection

441 (ATCC; Rockville, MD, USA) and cultured in Dulbecco's Modified Eagle Medium (DMEM)

442 supplemented with 10\% fetal bovine serum (FBS) and 1\% penicillin/streptomycin (Life technologies) at

$443 \quad 37^{\circ} \mathrm{C}$ with $5 \% \mathrm{CO} 2$. Cells (100000 cells/well in 6-well plates) were reverse-transfected in antibiotic-free

444 medium by $25 \mathrm{nM}$ miRNA mimic (ThermoFisher, 4464066) or control mimics (ThermoFisher, 4464058)

445 using Lipofectamine RNAiMAX Reagent (ThermoFisher,13778075) according to the manufacturer's

446 recommendations.

\section{RNA isolation and qRT-PCR analysis of miRNAs}

449 Total RNA was extracted using All Prep DNA/RNA/miRNA Universal kit (Qiagen) 48 hours after mimic 450 transfection. LightCycler 480 instrument (Roche) was used to perform qRT-PCR analysis of miR-29 and 451 miR-26 using TaqMan Fast Advanced miRNA Assays (ThermoFisher, 4444557) following guidelines 452 provided by the manufacturer. Expression was reported as $\mathrm{Ct}$ values (Fig. S5).

RNA-sequencing and analysis

455 Library preparation from total RNA was performed using NEB rRNA-depleted (HMR) stranded library 456 preparation kit according to manufacturer's instructions, and sequenced using Illumina NovaSeq 6000 457 (100bp paired-end). RNA-seq reads were aligned to the GRCh38 genome assembly using HISAT2 [53], 
and gene-level read counts were obtained using HTSeq-count [51] (“intersection-strict” mode) based on

459 Ensembl GRCh38 v87 gene annotations. DESeq2 [49] was used to compute differential gene expression

460 between cells expressing the miR-29 mimic and control mimic.

461

462 Declarations

\section{Ethics approval and consent to participate}

464 Not applicable.

465 Consent for publication

466 Not applicable.

467 Availability of data and materials

468 DiffRAC is available via GitHub at https://github.com/csglab/DiffRAC. Data generated during this study

469 are included in this published article and its supplementary files. Additional data and analysis files are

470 available at http://csg.lab.mcgill.ca/sup/pancancer_stability/ and via Zenodo

471 (doi:10.5281/zenodo.4404547). RNA-seq data from the miR-29 mimic expression experiment are

472 available via GEO under accession GSE145088. Other data used in this paper are available via their

473 source publications as indicated in the article.

474

475 Competing interests

476 The authors declare that they have no competing interests.

\section{$477 \quad$ Funding}

478 This work was supported by funds from Canadian Institutes of Health Research (PJT-155966), and

479 resource allocations from Compute Canada to H.S.N. H.S.N holds a Canada Research Chair funded by 
480 the Canadian Institutes of Health Research. G.P. and R.A. are supported by training scholarships from the

481 Canadian Institutes of Health Research, the Fonds de recherche du Québec - Santé (FRQS), and

482 Oncopole. Y.R. is a research scholar of the FRQS.

\section{Authors' contributions}

484 G.P. and H.S.N. conceived the study, developed the computational methods, analyzed the data, and wrote

485 the manuscript. P.J. and M.R. performed the miRNA-mimic experiment. R.A. contributed to data

486 processing. Y.R. contributed to experimental design and data interpretation. H.S.N. directed the study.

\section{Acknowledgements}

488 The results published here are in part based on data generated by the TCGA Research Network:

489 https://www.cancer.gov/tcga.

490 


\section{References}

1. Fish L, Navickas A, Culbertson B, Xu Y, Nguyen HCB, Zhang S, Hochman M, Okimoto R, Dill BD, Molina H, et al: Nuclear TARBP2 Drives Oncogenic Dysregulation of RNA Splicing and Decay. Mol Cell 2019, 75:967-981 e969.

2. Fish L, Zhang S, Yu JX, Culbertson B, Zhou AY, Goga A, Goodarzi H: Cancer cells exploit an orphan RNA to drive metastatic progression. Nat Med 2018, 24:1743-1751.

3. Goodarzi H, Liu X, Nguyen HC, Zhang S, Fish L, Tavazoie SF: Endogenous tRNA-Derived Fragments Suppress Breast Cancer Progression via YBX1 Displacement. Cell 2015, 161:790-802.

4. Goodarzi H, Nguyen HCB, Zhang S, Dill BD, Molina H, Tavazoie SF: Modulated Expression of Specific tRNAs Drives Gene Expression and Cancer Progression. Cell 2016, 165:14161427.

5. Perron G, Jandaghi P, Solanki S, Safisamghabadi M, Storoz C, Karimzadeh M, Papadakis AI, Arseneault M, Scelo G, Banks RE, et al: A General Framework for Interrogation of mRNA Stability Programs Identifies RNA-Binding Proteins that Govern Cancer Transcriptomes. Cell Rep 2018, 23:1639-1650.

6. Png KJ, Yoshida M, Zhang XH, Shu W, Lee H, Rimner A, Chan TA, Comen E, Andrade VP, Kim SW, et al: MicroRNA-335 inhibits tumor reinitiation and is silenced through genetic and epigenetic mechanisms in human breast cancer. Genes Dev 2011, 25:226-231.

7. Tavazoie SF, Alarcon C, Oskarsson T, Padua D, Wang Q, Bos PD, Gerald WL, Massague J: Endogenous human microRNAs that suppress breast cancer metastasis. Nature 2008, 451:147-152.

8. Vanharanta S, Marney CB, Shu W, Valiente M, Zou Y, Mele A, Darnell RB, Massague J: Loss of the multifunctional RNA-binding protein RBM47 as a source of selectable metastatic traits in breast cancer. Elife 2014, 3.

9. Goodarzi H, Najafabadi HS, Oikonomou P, Greco TM, Fish L, Salavati R, Cristea IM, Tavazoie S: Systematic discovery of structural elements governing stability of mammalian messenger RNAs. Nature 2012, 485:264-268.

10. Yang E, van Nimwegen, E., Zavolan, M., Rajewsky, N., Schroeder, M., Magnasco, M., \& Darnell, J. E., Jr: Decay rates of human mRNAs: correlation with functional characteristics and sequence attributes. Genome research 2003, 13:1863-1872.

11. Wada T, Becskei A: Impact of Methods on the Measurement of mRNA Turnover. Int J Mol Sci 2017, 18.

12. Schofield JA, Duffy EE, Kiefer L, Sullivan MC, Simon MD: TimeLapse-seq: adding a temporal dimension to RNA sequencing through nucleoside recoding. Nat Methods 2018, 15:221-225.

13. Blumberg A, Zhao Y, Huang Y-F, Dukler N, Rice EJ, Krumholz K, Danko CG, Siepel A: Characterizing RNA stability genome-wide through combined analysis of PRO-seq and RNA-seq data. 2019.

14. Lugowski A, Nicholson B, Rissland OS: Determining mRNA half-lives on a transcriptomewide scale. Methods 2018, 137:90-98.

15. Gaidatzis D, Burger L, Stadler MB: Analysis of intronic and exonic reads in RNA-seq data characterizes transcriptional and post-transcriptional regulation. Nat Biotechnol 2015, 33:722-729.

16. La Manno G, Soldatov R, Zeisel A, Braun E, Hochgerner H, Petukhov V, Lidschreiber K, Kastriti ME, Lonnerberg P, Furlan A, et al: RNA velocity of single cells. Nature 2018, 560:494-498.

17. Alkallas R, Fish L, Goodarzi H, Najafabadi HS: Inference of RNA decay rate from transcriptional profiling highlights the regulatory programs of Alzheimer's disease. Nat Commun 2017, 8:909. 
18. Gaidatzis D, Burger L, Florescu M, Stadler MB: Analysis of intronic and exonic reads in RNAseq data characterizes transcriptional and post-transcriptional regulation. Nat Biotechnol 2015, 33:722-729.

19. Zanzoni A, Spinelli L, Ribeiro DM, Tartaglia GG, Brun C: Post-transcriptional regulatory patterns revealed by protein-RNA interactions. Sci Rep 2019, 9:4302.

20. Joshi A, Van de Peer Y, Michoel T: Structural and functional organization of RNA regulons in the post-transcriptional regulatory network of yeast. Nucleic Acids Res 2011, 39:91089117.

21. Goodarzi H, Zhang S, Buss CG, Fish L, Tavazoie S, Tavazoie SF: Metastasis-suppressor transcript destabilization through TARBP2 binding of mRNA hairpins. Nature 2014, 513:256-260.

22. Ray D, Kazan H, Cook KB, Weirauch MT, Najafabadi HS, Li X, Gueroussov S, Albu M, Zheng $\mathrm{H}$, Yang A, et al: A compendium of RNA-binding motifs for decoding gene regulation. Nature 2013, 499:172-177.

23. Jonas S, Izaurralde E: Towards a molecular understanding of microRNA-mediated gene silencing. Nat Rev Genet 2015, 16:421-433.

24. Guo H, Ingolia NT, Weissman JS, Bartel DP: Mammalian microRNAs predominantly act to decrease target mRNA levels. Nature 2010, 466:835-840.

25. Lee JA, Damianov A, Lin CH, Fontes M, Parikshak NN, Anderson ES, Geschwind DH, Black DL, Martin KC: Cytoplasmic Rbfox1 Regulates the Expression of Synaptic and AutismRelated Genes. Neuron 2016, 89:113-128.

26. Weyn-Vanhentenryck SM, Mele A, Yan Q, Sun S, Farny N, Zhang Z, Xue C, Herre M, Silver PA, Zhang MQ, et al: HITS-CLIP and integrative modeling define the Rbfox splicingregulatory network linked to brain development and autism. Cell Rep 2014, 6:1139-1152.

27. Fogel BL, Wexler E, Wahnich A, Friedrich T, Vijayendran C, Gao F, Parikshak N, Konopka G, Geschwind DH: RBFOX1 regulates both splicing and transcriptional networks in human neuronal development. Hum Mol Genet 2012, 21:4171-4186.

28. Kanehisa M, Goto S: KEGG: kyoto encyclopedia of genes and genomes. Nucleic Acids Res 2000, 28:27-30.

29. Jopling C: Liver-specific microRNA-122: Biogenesis and function. RNA Biol 2012, 9:137-142.

30. Wu C, Zhang J, Cao X, Yang Q, Xia D: Effect of Mir-122 on Human Cholangiocarcinoma Proliferation, Invasion, and Apoptosis Through P53 Expression. Med Sci Monit 2016, 22:2685-2690.

31. Liu N, Jiang F, He TL, Zhang JK, Zhao J, Wang C, Jiang GX, Cao LP, Kang PC, Zhong XY, et al: The Roles of MicroRNA-122 Overexpression in Inhibiting Proliferation and Invasion and Stimulating Apoptosis of Human Cholangiocarcinoma Cells. Sci Rep 2015, 5:16566.

32. Ribatti D, Tamma R, Annese T: Epithelial-Mesenchymal Transition in Cancer: A Historical Overview. Transl Oncol 2020, 13:100773.

33. Meyer N, Penn LZ: Reflecting on 25 years with MYC. Nat Rev Cancer 2008, 8:976-990.

34. Dang CV: MYC on the path to cancer. Cell 2012, 149:22-35.

35. Warburg O, Wind F, Negelein E: The Metabolism of Tumors in the Body. J Gen Physiol 1927, 8:519-530.

36. Lal D, Pernhorst K, Klein KM, Reif P, Tozzi R, Toliat MR, Winterer G, Neubauer B, Nurnberg P, Rosenow F, et al: Extending the phenotypic spectrum of RBFOX1 deletions: Sporadic focal epilepsy. Epilepsia 2015, 56:e129-133.

37. Hu J, Ho AL, Yuan L, Hu B, Hua S, Hwang SS, Zhang J, Hu T, Zheng H, Gan B, et al: From the Cover: Neutralization of terminal differentiation in gliomagenesis. Proc Natl Acad Sci U S A 2013, 110:14520-14527.

38. Rajaram M, Zhang J, Wang T, Li J, Kuscu C, Qi H, Kato M, Grubor V, Weil RJ, Helland A, et al: Two Distinct Categories of Focal Deletions in Cancer Genomes. PLoS One 2013, 8:e66264. 
39. Andersen CL, Lamy P, Thorsen K, Kjeldsen E, Wikman F, Villesen P, Oster B, Laurberg S, Orntoft TF: Frequent genomic loss at chr16p13.2 is associated with poor prognosis in colorectal cancer. Int J Cancer 2011, 129:1848-1858.

40. Huang YT, Heist RS, Chirieac LR, Lin X, Skaug V, Zienolddiny S, Haugen A, Wu MC, Wang Z, $\mathrm{Su} \mathrm{L}$, et al: Genome-wide analysis of survival in early-stage non-small-cell lung cancer. $J$ Clin Oncol 2009, 27:2660-2667.

41. Monteith GR, Prevarskaya N, Roberts-Thomson SJ: The calcium-cancer signalling nexus. Nat Rev Cancer 2017, 17:367-380.

42. Shen F, Xu X, Yu Z, Li H, Shen H, Li X, Shen M, Chen G: Rbfox-1 contributes to CaMKIIalpha expression and intracerebral hemorrhage-induced secondary brain injury via blocking micro-RNA-124. J Cereb Blood Flow Metab 2020:271678X20916860.

43. He H, Wang L, Zhou W, Zhang Z, Wang L, Xu S, Wang D, Dong J, Tang C, Tang H, et al: MicroRNA Expression Profiling in Clear Cell Renal Cell Carcinoma: Identification and Functional Validation of Key miRNAs. PLoS One 2015, 10: 0125672.

44. Yan B, Guo Q, Fu FJ, Wang Z, Yin Z, Wei YB, Yang JR: The role of miR-29b in cancer: regulation, function, and signaling. Onco Targets Ther 2015, 8:539-548.

45. Park SY, Lee JH, Ha M, Nam JW, Kim VN: miR-29 miRNAs activate p53 by targeting p85 alpha and CDC42. Nat Struct Mol Biol 2009, 16:23-29.

46. Heinzelmann J, Henning B, Sanjmyatav J, Posorski N, Steiner T, Wunderlich H, Gajda MR, Junker K: Specific miRNA signatures are associated with metastasis and poor prognosis in clear cell renal cell carcinoma. World J Urol 2011, 29:367-373.

47. Garzon R, Heaphy CE, Havelange V, Fabbri M, Volinia S, Tsao T, Zanesi N, Kornblau SM, Marcucci G, Calin GA, et al: MicroRNA 29b functions in acute myeloid leukemia. Blood 2009, 114:5331-5341.

48. Sengupta S, den Boon JA, Chen IH, Newton MA, Stanhope SA, Cheng YJ, Chen CJ, Hildesheim A, Sugden B, Ahlquist $P$ : MicroRNA 29c is down-regulated in nasopharyngeal carcinomas, up-regulating mRNAs encoding extracellular matrix proteins. Proc Natl Acad Sci US A 2008, 105:5874-5878.

49. Love MI, Huber W, Anders S: Moderated estimation of fold change and dispersion for RNAseq data with DESeq2. Genome Biol 2014, 15:550.

50. Wang L, Wang S, Li W: RSeQC: quality control of RNA-seq experiments. Bioinformatics 2012, 28:2184-2185.

51. Anders S, Pyl PT, Huber W: HTSeq--a Python framework to work with high-throughput sequencing data. Bioinformatics 2015, 31:166-169.

52. Liberzon A, Birger C, Thorvaldsdottir H, Ghandi M, Mesirov JP, Tamayo P: The Molecular Signatures Database (MSigDB) hallmark gene set collection. Cell Syst 2015, 1:417-425.

53. Kim D, Paggi JM, Park C, Bennett C, Salzberg SL: Graph-based genome alignment and genotyping with HISAT2 and HISAT-genotype. Nat Biotechnol 2019, 37:907-915.

54. Agarwal V, Bell GW, Nam JW, Bartel DP: Predicting effective microRNA target sites in mammalian mRNAs. Elife 2015, 4.

55. Subramanian A, Tamayo P, Mootha VK, Mukherjee S, Ebert BL, Gillette MA, Paulovich A, Pomeroy SL, Golub TR, Lander ES, Mesirov JP: Gene set enrichment analysis: a knowledgebased approach for interpreting genome-wide expression profiles. Proc Natl Acad Sci U S A 2005, 102:15545-15550.

56. Pinto JP, Kalathur RK, Oliveira DV, Barata T, Machado RS, Machado S, Pacheco-Leyva I, Duarte I, Futschik ME: StemChecker: a web-based tool to discover and explore stemness signatures in gene sets. Nucleic Acids Res 2015, 43:W72-77. 
a

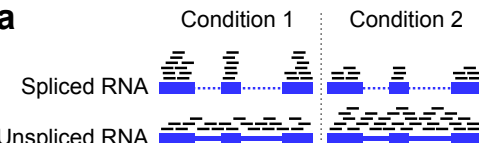

- Slower transcription - Faster transcription - Higher stability - Lower stability

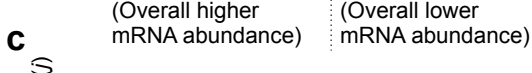

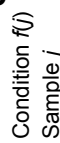
mRNA abundance) mRNA abundance)

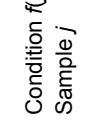

ఖ 1 1 1 :

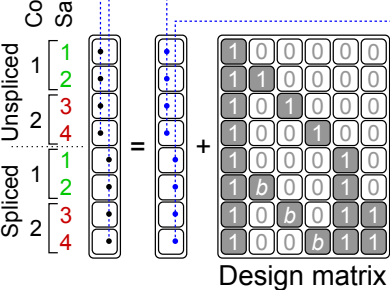

Design matrix

e

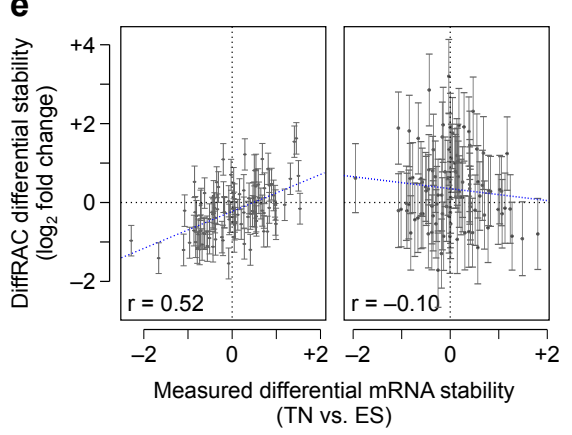

b Mean read count for gene $i$ in sample $j$ Abundance for gene $i$ in sample $j$ Gene-specific scaling factor Sample-specific scaling factor Intronic $\cdots\left[\lambda_{i, j}^{\text {int }}=p_{i, j} \times l_{i} \times s_{j}^{\text {int }} \quad \begin{array}{l}\text { mRNA stability for gene } i \text { as a } \\ \text { function of the condition } f(j)\end{array}\right.$ Exonic $\cdots . . .\left[\lambda_{i, j}^{e x o}=m_{i, j} \times l_{i}^{\prime} \times s_{j}^{e x o} \quad m_{i, j}=\left(p_{i, j}\right)^{b} \times \stackrel{\square}{\gamma_{i, f(j)}}\right.$

d

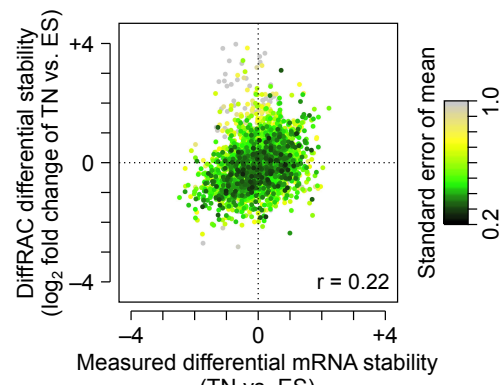
(TN vs. ES)

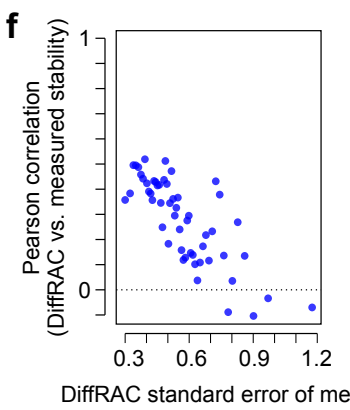

(average for bins of 50 genes)

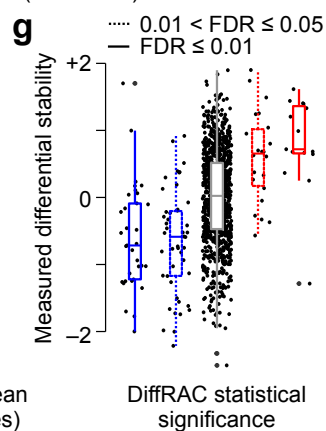

641 Figure 1. Inference of differential mRNA stability using DiffRAC. (a) Schematic representation of the effect of transcription and stability on the abundances of unspliced and spliced RNA. (b) DiffRAC models the mean $(\lambda)$ of intronic (int) and exonic (exo) read distribution as a function of pre-mature $(p)$ and mature $(m)$ transcript abundances, in addition to gene-specific $(l)$ and library-specific $(s)$ scaling factors. The mature mRNA abundance is itself modeled as a function of the pre-mature RNA abundance and mRNA stability $(\gamma)$, which is in turn a function $(f)$ of the experimental variables. Also see Fig. S1. (c) An example case with four samples and two experimental conditions, showing how DiffRAC's model can be implemented in a regression with a log-link function, along with the interpretation of regression coefficients (also see Methods). (d) Comparison of DiffRAC stability estimates against experimental mRNA half-life (stability) measurements in mouse ES cells differentiated to terminal neurons (TN) [18]. Each data point stands for one gene, with the points colored according the standard error of the mean (SEM) for DiffRAC estimates. (e) Comparison of DiffRAC estimates vs. measured stability for the 100 genes with the smallest (left) and largest (right) DiffRAC SEMs. Error bars represent SEM. (f) The Pearson correlation between DiffRAC estimates and measured stability for bins of 50 genes sorted by their SEM. (g) Distribution of experimental half-life measurements for genes that DiffRAC has identified as significantly destabilized (blue boxplots) or stabilized (red boxplots) in TN vs. ES cells, at FDR cutoffs of 0.05 (dashed line) or 0.01 (solid line). Genes that are 

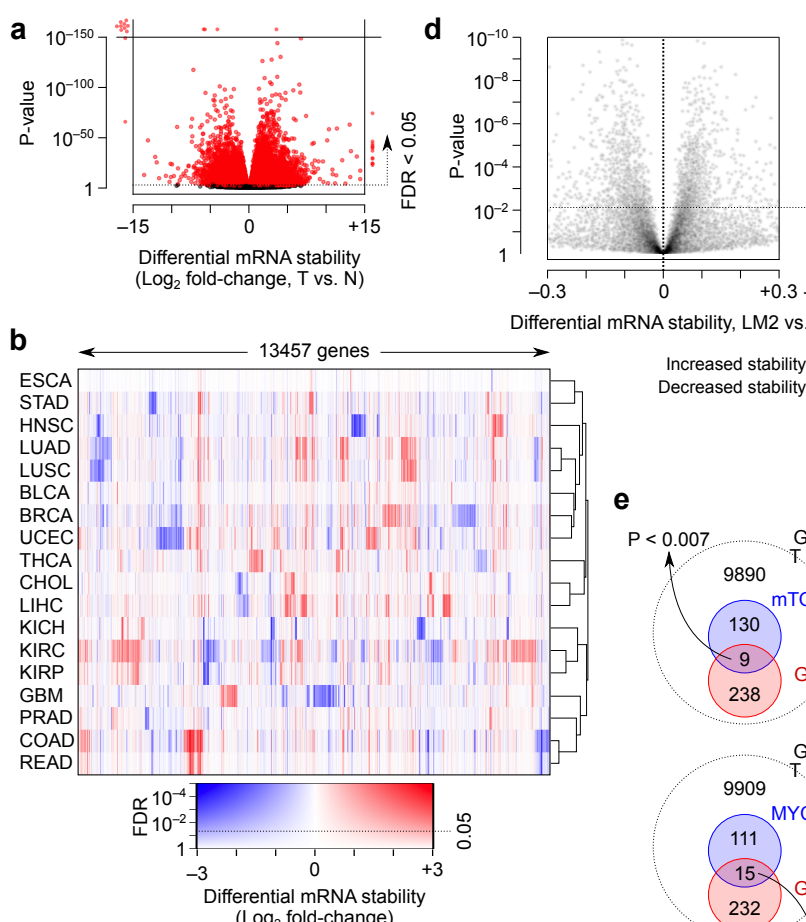

( Log $_{2}$ fold-change)

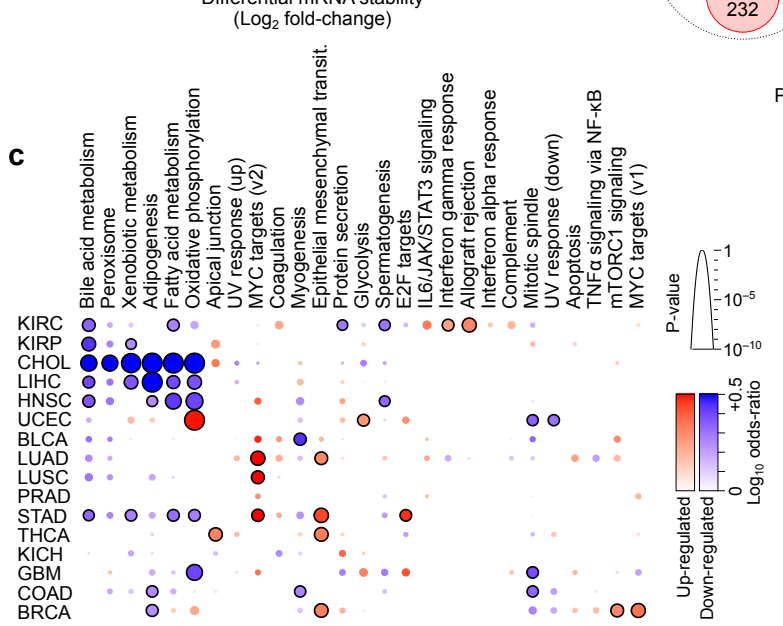

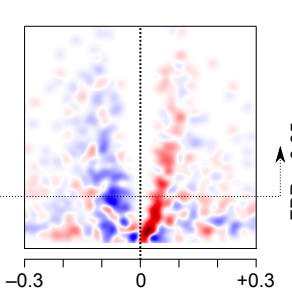

Stage-associated stability changes

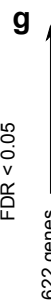

$P<0.007 \quad$ Genes measured in both 9890 T vs. N and LM2 vs. MDA

$130{ }^{\text {mTORC1 signaling }}$ 19
238
Geres significantly stabilized in
T vs. N and in LM2 vs. MDA Genes measured in both
T vs. N and LM2 vs. MDA 9909 MYC targets v1

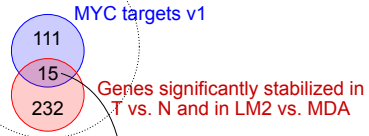
(1) $P<4 \times 10^{-7}$

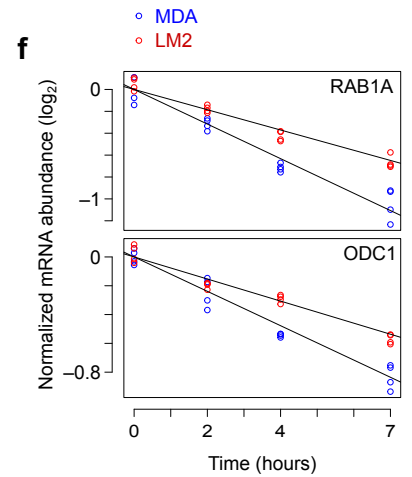

h $\quad \begin{aligned} & \mathrm{LIHC} \\ & r=0.28 \pm 0.02 \\ & r=0.66 \pm 0.16\end{aligned}$

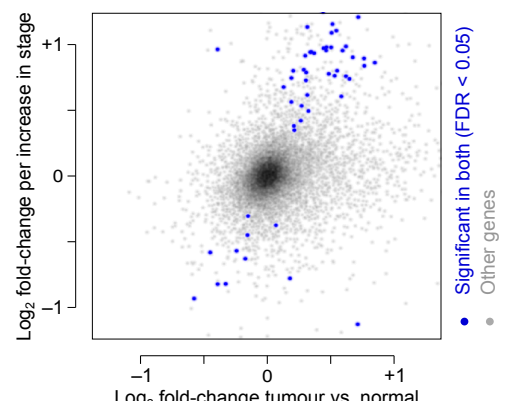

$\log _{2}$ fold-change tumour vs. normal i

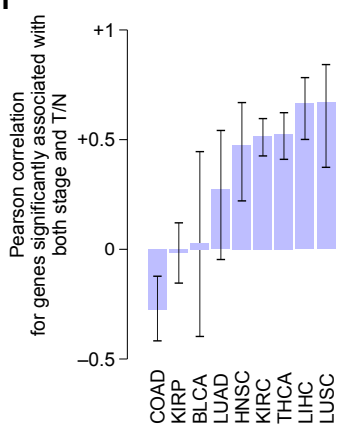

Figure 2. Pan-cancer analysis of differential mRNA stability. (a) Volcano plot of differential RNA stability between tumour and normal tissues for 18 TCGA cancer types. See Fig. S3 for volcano plots of individual cancer types. (b) Heatmap of differential mRNA stability profiles across TCGA cancers. Genes with significant DiffRAC results in at least one cancer $\left(\mathrm{FDR}<0.05\right.$ ) are included. The color gradient represents a combination of the $\log _{2}$ fold-change of mRNA stability and the FDR. BLCA: bladder urothelial carcinoma, BRCA: breast invasive carcinoma, CHOL: cholangiocarcinoma, COAD: colon adenocarcinoma, ESCA: esophageal carcinoma, GBM: glioblastoma multiforme, HNSC: head and neck squamous cell carcinoma, KICH: kidney chromophobe, KIRC: kidney renal clear cell carcinoma, KIRP: kidney renal papillary cell carcinoma, LIHC: liver hepatocellular carcinoma, LUAD: lung adenocarcinoma, LUSC: lung squamous cell carcinoma, PRAD: prostate adenocarcinoma, READ: rectum adenocarcinoma, STAD: stomach adenocarcinoma, THCA: thyroid carcinoma, UCEC: uterine corpus endometrial carcinoma. (c) Pathway enrichment analysis of genes with significant differential mRNA stability in each cancer type. Circles with black outline correspond to MSigDB hallmark gene sets that are significantly enriched among cancer-stabilized (red) and cancer-destabilized (blue) genes (FDR $<0.05$, Fisher's exact test). Log-odds and P-values are represented using the color gradient and circle sizes, respectively. (d) The 
672 volcano plot on the left shows the experimentally measured differential stability between highly metastatic MDA-

673 LM2 cell line relative to its parental MDA-MB-231 line (see Methods). The right panel shows the relative

674 enrichment of genes that were stabilized (red) or destabilized (blue) in TCGA-BRCA tumors compared to normal

675 samples. Kernel density estimation was used to calculate the density of BRCA-stabilized and destabilized genes

676 across the plot, with the difference between the estimated densities of the two groups shown using the color

677 gradient. (e) Venn diagrams illustrating the overlap between genes that are significantly stabilized in BRCA tumors

678 (relative to normal) and MDA-LM2 (relative to MDA-MB-231), and genes that are part of the mTORC1 signalling

679 (top) or MYC targets (bottom). P-values are based on Fisher's exact test. (f) Transcription inhibition time course

680 graphs for two example genes, one involved in mTORC1 signaling (RAB1A) and one among MYC targets (ODC1).

681 Time course measurements in MDA-LM2 and parental MDA-MB-231 cells are shown in red and blue, respectively,

682 with the slope of each fitted line representing the rate of degradation. (g) Stability changes associated with tumor

683 stage (left) and grade (right) across TCGA cancers. Genes with significant changes in at least one cancer at FDR <

6840.05 are included. The color gradient is the same as in panel b. (h) Comparison of the differential mRNA stability

685 between tumour and normal (x-axis) and stage-associated differential mRNA stability (y-axis), in the TCGA-LIHC

686 dataset as an example. Genes with significant changes along both axes at FDR $<0.05$ are colored in blue. Pearson

687 correlation coefficients and confidence intervals for all genes (black) and significant ones (blue) are shown on top.

688 Panel (i) summarizes the Pearson correlations for significant genes in other cancer types (error bars represent the

689 confidence intervals). 
a

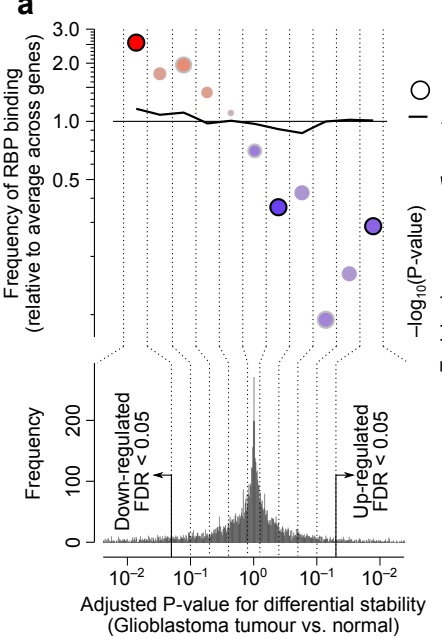

C

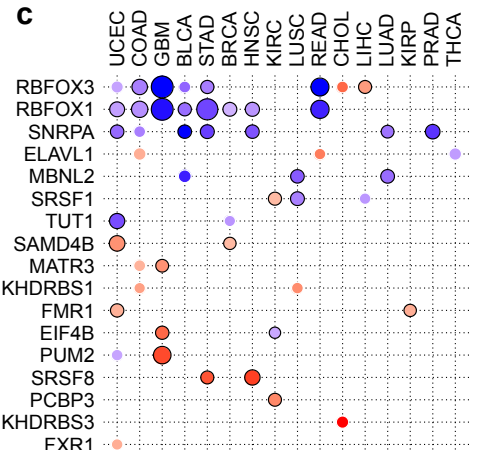

b

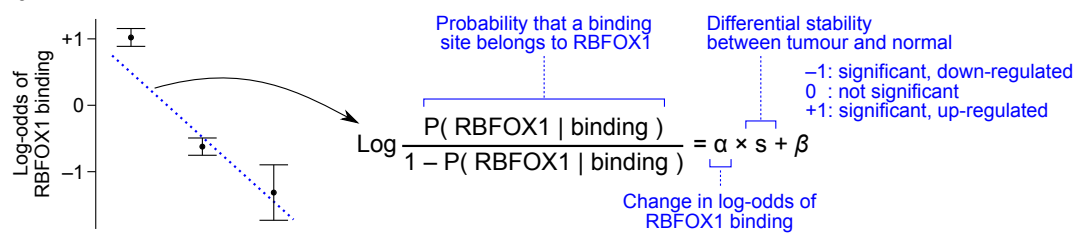

SNRPA - BLCA SNRPA - PRAD ELAVL1 - READ RBFOX3 - LIHC SNRPA - UCEC SAMD4B - BRCA MATR3 - COAD KHDRBS1 - COAD SNRPA - COAD ELAVL1 - COAD SNRPA - LUAD KHDRBS1 - LUSC TUT1 - UCEC PUM2 - GBM SNRPA - HNSC RBFOX3 - CHOL EIF4B - GBM SAMD4B - UCEC MATR3 - GBM TUT1 - BRCA SRSF - LIHC

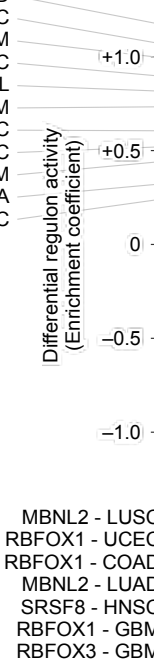
SRSF1 - LUSC
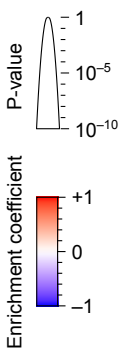

Figure 3: Enrichment of RBP binding sites among differentially stabilized genes in cancer. (a) An example case showing the enrichment of RBFOX1 binding sites among differentially stabilized genes in TCGA-GBM. Genes are binned by FDR of their DiffRAC differential stability between tumour and normal, with destabilized genes on the left and stabilized genes on the right. The relative frequency of RBFOX1 targets (circles) and targets of all other RBPs (solid line) is shown for each bin. (b) Schematic representation of the logistic regression approach for modeling the enrichment of RBFOX1 targets (relative to other RBPs) as a function of differential stability. (c) Heatmap summarizing the results of applying the model in panel $\mathbf{b}$ to all RBPs. Positive (red) and negative (blue) regression coefficients indicate enrichment of RBP targets among genes that are stabilized and destabilized in cancer, respectively. The circle size represents the significance level. Significant associations between RBP binding and stability status are shown using black outlines (FDR $<0.05$ ). (d) Comparison of the differential RBP expression (tumor vs normal) and cancer-associated regulon activity. Regulon activity is defined to be the same as the enrichment coefficients from panel $\mathbf{c}$, with the sign of the coefficient inverted for RBPs whose binding leads to RNA destabilization (based on [22]). Each dot represents one RBP in one cancer type. Pearson correlation of differential expression vs. differential regulon activity is 0.61 . 
a

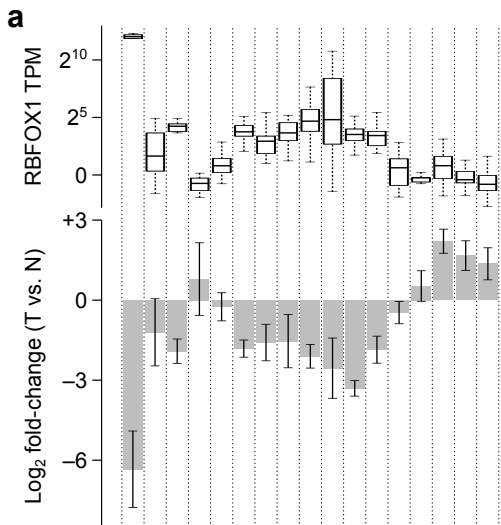

b

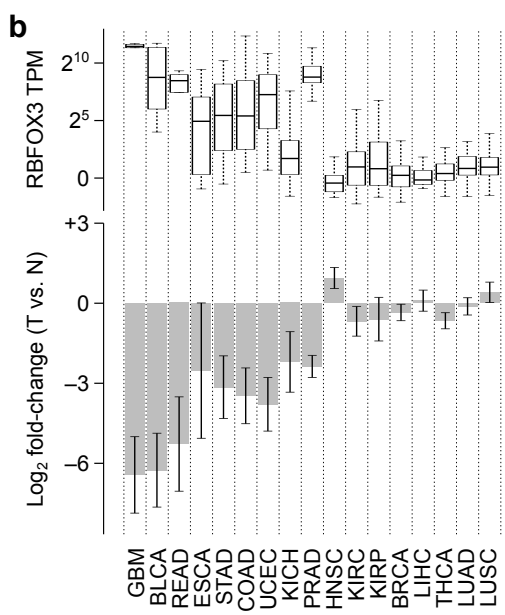

c

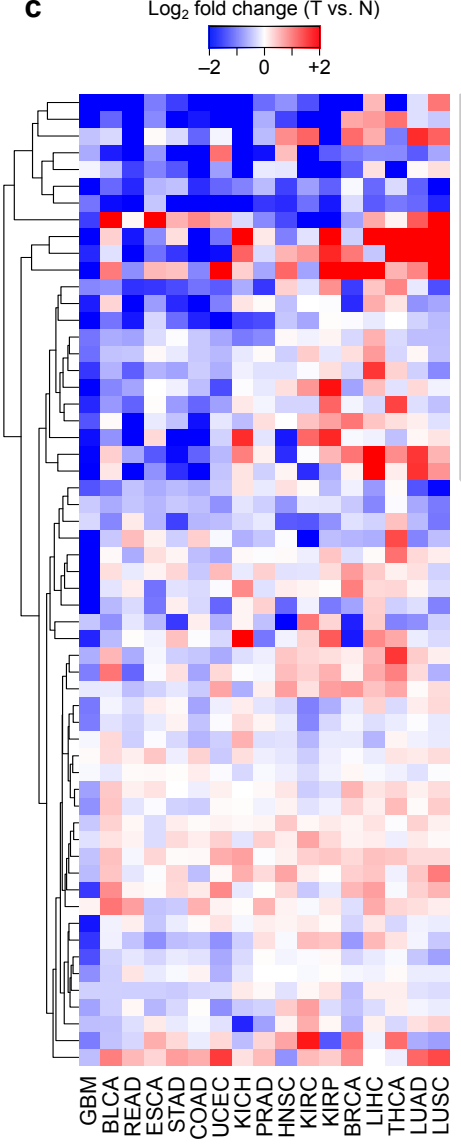

d
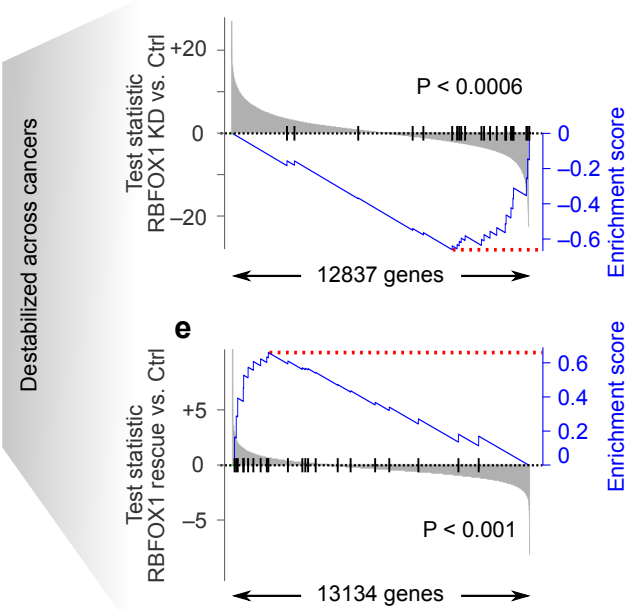

f
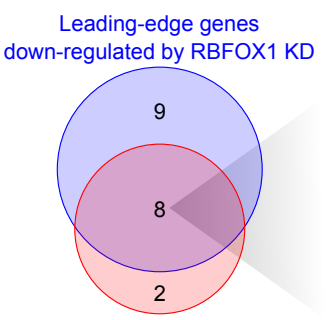

up-regulated by RBFOX1 rescue

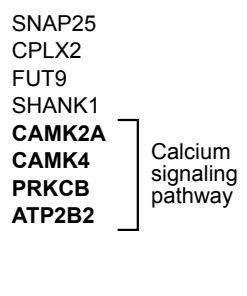

Figure 4: Aberrant activity of RBFOX proteins mediates stability changes across multiple cancers. (a) RBFOX1 expression across TCGA cancer types. The box plot (top) shows the RBFOX1 transcript-per-million (TPM) values in normal tissue samples. The bar plot (bottom) illustrates the average log fold-change of RBFOX1 expression in tumors compared to normal samples (error bars represent SEM). (b) RBFOX3 expression in normal tissue samples and differential expression in tumors, similar to panel a. (c) Heatmap showing the stability of RBFOX HITS-CLIP targets. HITS-CLIP targets include genes that have a mouse homolog with a strong RBFOX peak (height 2200 ) in 3' UTR, as defined previously [26]. Rows correspond to genes and columns to cancer types, with the latter sorted in the same order as panels a-b. (d) Gene set enrichment analysis (GSEA) [55] for RBFOX1 inhibition in terminally differentiated neurons. Genes (x-axis) are sorted by the Wald test statistic of differential expression between RBFOX1 knockdown and control cells, with vertical black lines demarcating the pan-cancerdestabilized set of RBFOX1 targets. The blue line represents the enrichment curve for this gene set [55]. (e) GSEA for RBFOX1 rescue in mouse neurons deficient for RBFOX proteins, similar to panel d. (f) Venn diagram illustrating the overlap between the leading-edge [55] set of genes downregulated by RBFOX1 knockdown (from d) and the leading-edge set of genes upregulated by RBFOX1 rescue (from e). 

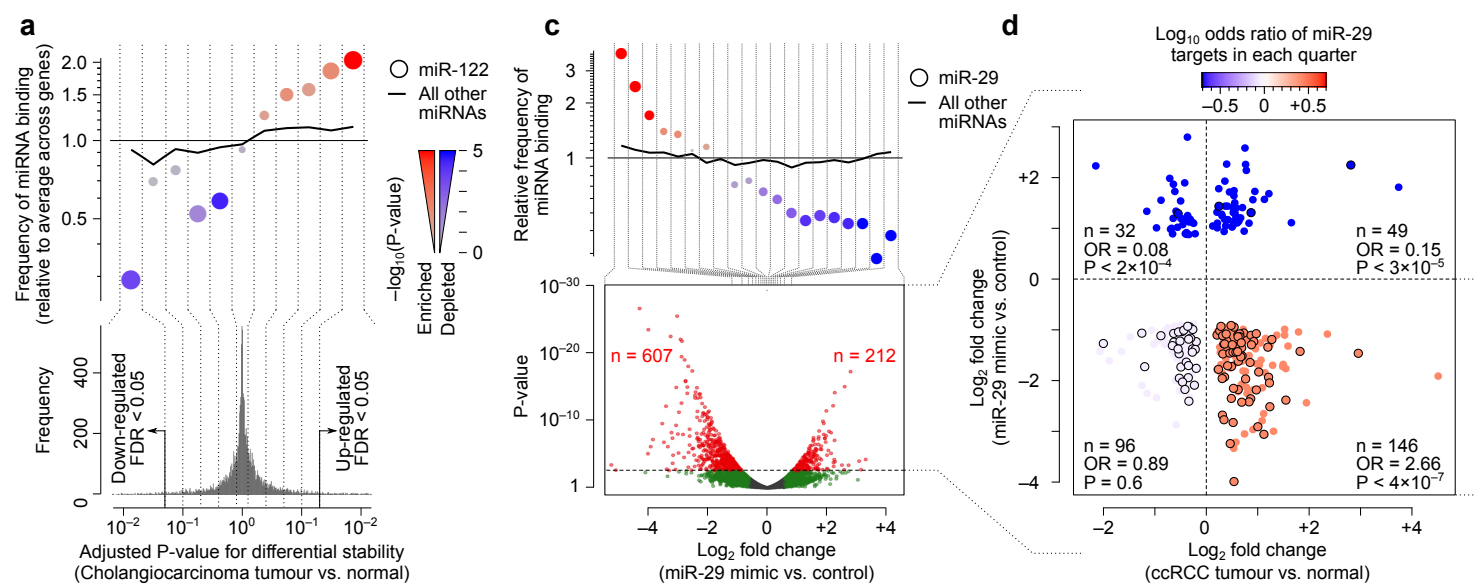

e
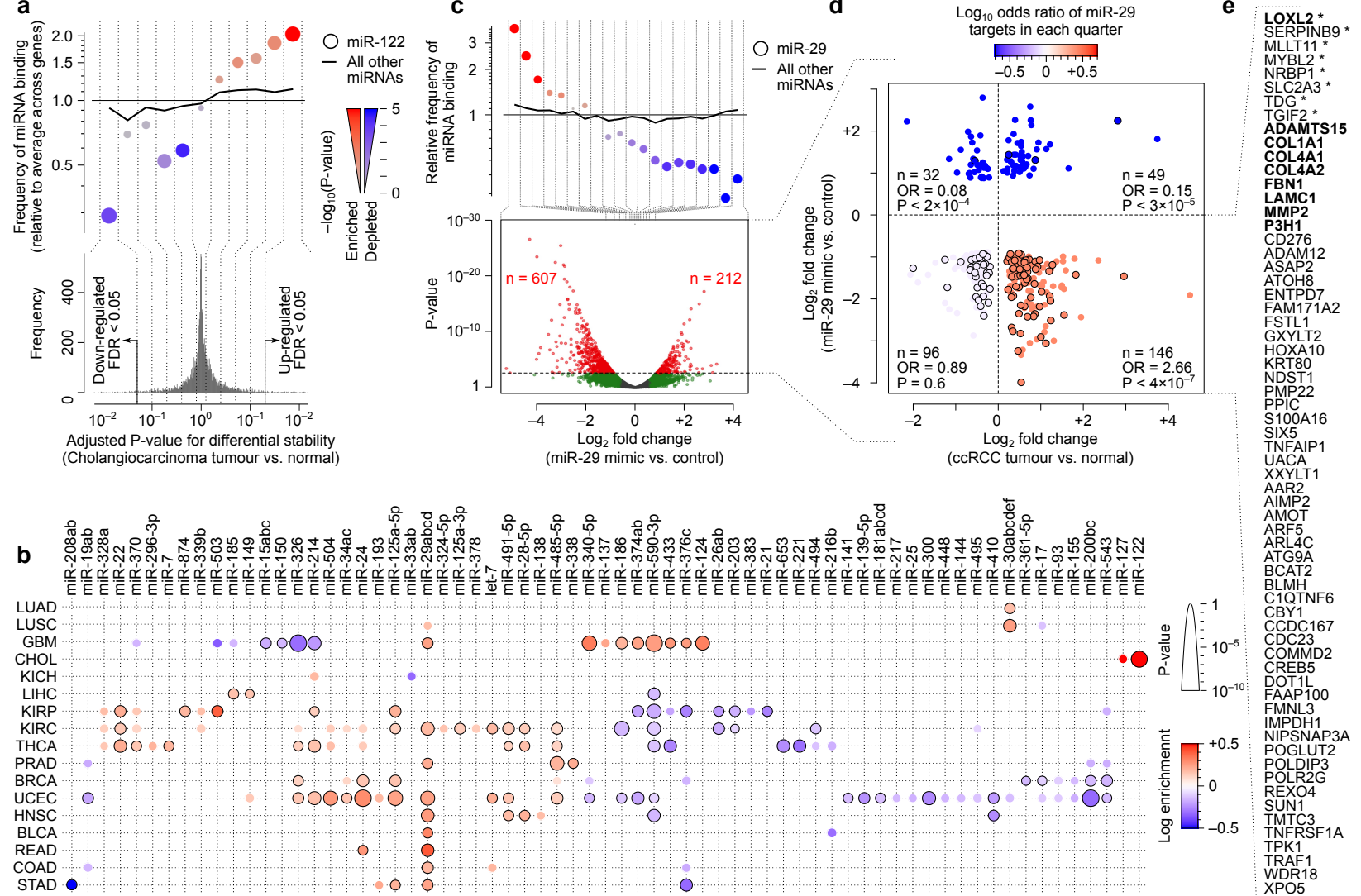

Figure 5: Dysregulation of miRNA regulons in cancer. (a) An example case showing the enrichment of miR-122 targets among genes stabilized in TCGA-CHOL tumours (relative to normal), similar to Fig. 3a. Note that since miRNAs are expected to destabilize their targets, enrichment in differentially stable genes indicates down-regulation of miRNA activity. (b) Heatmap summarizing enrichment analysis for all miRNAs across all cancer types, similar to Fig. 3c. (c) Enrichment of miR-29 targets among genes that are destabilized after transfection of miR-29 mimic in 786-O cells. The volcano plot (bottom) summarizes differential expression results between miR-29 mimic and control; the dot plot at the top shows enrichment of miR-29 targets at bins of differentially expressed genes, similar to panel a. In the volcano plot, significantly differentially expressed genes (FDR $<0.05)$ are shown in red. (d) Enrichment of miR-29 binding sites, relative to other miRNA binding sites, in genes categories defined by their differential stability in TCGA-KIRC and differential expression after miR-29 mimic expression in 786-O cells. Each dot represents a gene, and those with a black outline contain at least one miR-29 binding site. The color gradient represents the log-odds of miR-29 binding site enrichment in each quarter. P-values are based on Fisher's exact test. (e) List of genes that are bound by miR-29, up-regulated in KIRC, and down-regulated after miR-29-mimic treatment of 786-O cells. Genes that are bold correspond to ECM genes (based on overlap with GO; enrichment P < $6 \times 10^{-6}$ ), and those with an asterisk are markers of embryonal carcinoma (based on StemCheker [56]; enrichment P $<3 \times 10^{-4}$ ). 


\section{Description of Supplementary Files}

740 Supplementary Figures: This document includes Figures S1-S6, their associated figure legends, and

741 references that are cited within supplementary figure legends.

742 Table S1: Estimation of differential mRNA stability using the RNA-seq data from mouse embryonic

743 stem cells and terminal neurons, along with ground-truth transcript half-life measurements after

744 transcriptional blockage with actinomycin D. DiffRAC estimates are listed in columns 2-7. Ground-truth

745 half-life measurements are in column 8.

746 Table S2: Pan-cancer analysis of differential mRNA stability across TCGA cancers, comparing tumors to

747 normal tissue. DiffRAC estimates for each cancer type are provided in separate sheets.

748 Table S3: Differential stability comparing the MDA-MB-231 and MDA-LM cell lines.

749 Table S4: Clinical data and tumor purity for TCGA samples that were used for analysis of stage- and/or 750 grade-associated stability changes.

751 Table S5: Genes with significant stability changes associated with tumor stage in at least one of the 11

752 TCGA cancers for which this type of classification was available. Note that no significant associations 753 were identified for READ, and therefore this cancer type has no entries in this table.

754 Table S6: Genes with significant stability changes associated with tumor grade in at least one of the four cancer types for which this type of classification was available.

Table S7: Differential gene expression in RBFOX1 knockdown in terminally differentiated neurons.

DESeq2 estimates are provided.

Table S8: Differential gene expression in the miR-29 mimic relative to control mimic expression in 786- 